\title{
Antigen Capture Enzyme-Linked Immunosorbent Assay for Detecting Middle East Respiratory Syndrome Coronavirus in Humans
}

\author{
Joshua Fung, Susanna K. P. Lau, and Patrick C. Y. Woo
}

\begin{abstract}
The Middle East respiratory syndrome (MERS) is the second novel zoonotic disease infecting humans caused by coronavirus $(\mathrm{CoV})$ in this century. To date, more than 2200 laboratory-confirmed human cases have been identified in 27 countries, and more than 800 MERS-CoV associated deaths have been reported since its outbreak in 2012. Rapid laboratory diagnosis of MERS-CoV is the key to successful containment and prevention of the spread of infection. Though the gold standard for diagnosing MERS-CoV infection in humans is still nucleic acid amplification test (NAAT) of the up-E region, an antigen capture enzymelinked immunosorbent assay (ELISA) could also be of use for early diagnosis in less developed locations. In the present method, a step-by-step guide to perform a MERS-CoV nucleocapsid protein (NP) capture ELISA using two NP-specific monoclonal antibodies is provided for readers to develop their in-house workflow or diagnostic kit for clinical use and for mass-screening project of animals (e.g., dromedaries and bats) to better understand the spread and evolution of the virus.
\end{abstract}

Key words MERS-CoV, Nucleocapsid protein, Molecular detection, Clinical diagnosis, Antigen capture ELISA, Immunoassay

\section{Introduction}

The Middle East respiratory syndrome (MERS) is the second novel zoonotic disease infecting humans caused by coronavirus $(\mathrm{CoV})$ in this century. To date, more than 2200 laboratory-confirmed human cases have been identified in 27 countries, and more than 800 MERS-CoV associated deaths have been reported since its outbreak in 2012 [1]. Rapid laboratory diagnosis of MERS-CoV is the key to successful containment and prevention of the spread. Nucleic acid amplification test (NAAT, e.g., real-time reverse transcription quantitative polymerase chain reaction [real-time RT-qPCR]), virus isolation, transmission electron microscopy, immunohistochemistry, and serological methods (e.g., antigen capture enzyme-linked immunosorbent assay [ELISA] and 
immunofluorescence assay [IFA]) have been developed and used for MERS-CoV diagnosis [2-7]. While the "gold standard" for MERS-CoV diagnosis is NAAT of the upper region of the envelope gene (up-E) or the nucleocapsid $(\mathrm{N})$ gene as suggested by the World Health Organization (WHO), antigen capture ELISA assay for MERS-CoV can also be informative when NAAT is not available or when the serological assay is used to confirm the findings and aid treatment decision $[2,3]$.

Further to diagnosing possible human infection of MERS$\mathrm{CoV}$, this method is also useful for screening the virus in the wildlife or agricultural applications. Government agencies and research groups may find serological tests like antigen capture ELISA to be more economical than NAATs for routine screening of MERS-CoV in farm-held or city-dwelling animals. The antigen capture ELISA described in this method offers four significant advantages over traditional NAATs.

Firstly, serological screening requires less space in facilities and can be performed in point-of-care locations to minimize sample transporting and reduce turnover time. To avoid crosscontamination from amplicons in NAATs, the workflow usually requires four separate physical locations: (1) sample preparation (lysis, extraction of nucleic acids, and reverse transcription), (2) NAAT master mix preparation, (3) template addition, and (4) amplification and analysis. Though technologies like real-time RT-qPCR simplify the workflow, such requirements limit the assay to be performed in regional laboratories designed or designated for this application. Antigen capture ELISA, on the other hand, can be performed on open bench in a single location after virus inactivation, allowing it to be performed in even the most minimally designed facility.

Secondly, antigen capture ELISA can be performed with simple equipment and can be established with limited initial investment. For performing NAATs at a modern standard, UV cabinets or workstations for master mix preparation and sample addition, thermal cyclers, agarose gel running, and visualization equipment are the least requirement. For more stringent testing and faster turnaround, it calls for a real-time PCR thermal cycler (e.g., Roche's LightCycler systems or Bio-Rad Touch detection systems) which requires a fair amount of initial investment and limits the assay from being performed in remote or less developed locations. In contrast, antigen capture ELISA and other serological methods can be performed with much simpler equipment. Multichannel pipettes, automatic plate washer, and plate reader are the only specialized tools needed for this application and can be purchased with ease if those are not already available.

Thirdly, much less training is required for technicians to handle serological testing than NAATs. Though NAATs and ELISA are some of the most basic assays performed in a medical laboratory 
and minimal training is needed for an experienced worker to perform such task, to allow quicker and broader surveillance of MERS$\mathrm{CoV}$ in human and animal population, it would be beneficial to set up more surveillance facilities in the less developed parts of the world. The time and resources needed to train a novice laboratory worker to perform ELISA are much less, as only dilution and pipetting skills are required.

Fourthly, common nucleic staining chemicals used in NAATs for amplicon visualization are a possible mutagen and post potential health risk to workers and the surrounding environment; while chemicals and solutions used in ELISA are relatively safer. To visualize the amplicons after agarose gel electrophoresis or during the qPCR thermal cycles, dyes like ethidium bromide (EtBr), SYBR Green, or Gel Red are used; while EtBr is a known mutagen, others are a relatively new addition to the market and extensive safety data is not widely available [8]. In comparison, the chemicals and solutions used in ELISA are commonly found in clinical and research laboratories and are generally safe when used properly.

Finally, and most importantly, antigen capture ELISA can offer high sensitivity and specificity for MERS-CoV diagnosis in even early infection and animal samples. We have previously demonstrated that by using two MERS-CoV nucleocapsid protein (NP) specific monoclonal antibodies (MAbs) in performing capture ELISA, the test can accurately detect MERS-CoV virus down to $10 \mathrm{TCID}_{50} / 0.1 \mathrm{~mL}$ and has a specificity of $100 \%$ [3]. As the nasopharyngeal aspirate viral load from patients during acute infection are around $10^{6}$ copies $/ \mathrm{mL}$ and nasal samples in dromedaries are usually around $10^{4}-10^{6}$ copies $/ \mathrm{mL}$, this test offers sufficient sensitivity for MER-CoV diagnosis and screening [9-11].

Other forms of MERS-CoV serological diagnostic test have also been developed based on different principles and are designed to fulfill different purposes, one should also review those options and evaluate their needs. To detect seroconversion from previous infection of MERS-CoV, the WHO suggests laboratories to perform IFA or ELISA together with neutralization assay, the result alone can be used to determine if it is a confirmed case, regardless of the results from NAAT assay [2]. For rapid on-site diagnosis of MERS-CoV, we have previously reported the adaptation of the antigen capture ELISA in the format of lateral flow immunoassay (LFIA). This assay can yield results in under half an hour, requires minimal equipment, training, and can be stored at room temperature, thus allowing it to be performed in the field [12]. This LFIA is also able to detect MERS-CoV-like viruses (e.g., Tylonycteris bat CoV HKU4 and Pipistrellus bat CoV HKU5) and is useful for the research to understand the evolutional history of MERS-CoV $[13,14]$.

In the current manuscript, the method for performing NP capture ELISA using two MERS-CoV-NP-specific monoclonal 


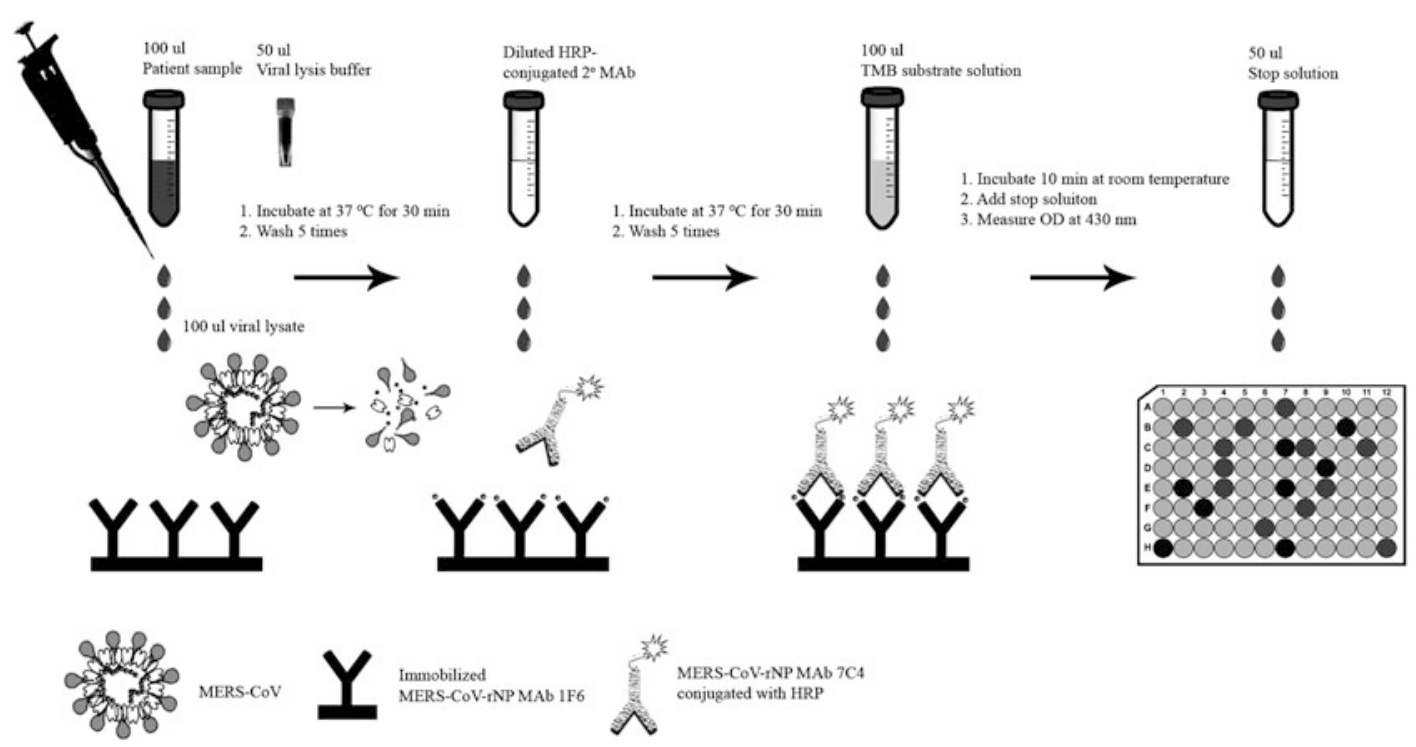

Fig. 1 Schematic diagram showing the general workflow of the MERS-CoV NP antigen capture ELISA

antibodies (MAbs) will be introduced. The general workflow of the assay is summarized in a figure for quick referencing [3, 15] (Fig. 1).

\section{Materials}

\subsection{Reagents and} Equipment
1. $1.5 \mathrm{~mL}$ conical screw cap tubes.

2. 10, 200, 300 (optional), and $1000 \mu \mathrm{L}$ filtered pipette tips.

3. Single-channel (covering $10-1000 \mu \mathrm{L})$ and 8 -channel $(200 \mu \mathrm{L}$ or $300 \mu \mathrm{L}$; optional) pipettes.

4. 96-Well high binding microtiter plates or strips with holder for ELISA.

5. Adhesive sealing film for microtiter plates.

6. $50 \mathrm{~mL}$ solution reservoir for multichannel pipettes.

7. Automated microtiter plate washer-dispenser (able to handle 96-well plates and microwell strips; optional) (see Note 1).

8. Microtiter plate spectrophotometer able to read optical density (OD) at $450 \mathrm{~nm}$.

9. Platform rocker.

10. Two purified MERS-CoV NP MAbs with nonoverlapping epitopes.

11. TMB solution.

12. MAb 7C4 conjugated with HRP.

13. $3,3^{\prime}, 5,5^{\prime}$-Tetramethylbenzidine (TMB) substrate solution. 
2.2 Buffers

\section{Methods}

\subsection{Designing the Assay}

1. Phosphate-buffered saline (PBS): $144 \mathrm{mg}$ potassium phosphate monobasic, $9000 \mathrm{mg}$ sodium chloride, and $795 \mathrm{mg}$ of sodium phosphate dibasic salts in $1 \mathrm{~L}$ of water.

2. Washing buffer: PBS containing 0.5\% Tween 20 .

3. Blocking buffer: PBS containing $2 \%$ sucrose, $0.2 \%$ casein- $\mathrm{Na}$, and $2 \%$ gelatin.

4. Enzyme dilution buffer: PBS containing 0.5\% Tween 20 and $20 \%$ fetal calf serum.

5. Sample dilution buffer: PBS containing $2 \%$ skim milk.

6. Stop solution: $0.2 \mathrm{M}$ sulfuric acid.

7. Viral Lysis Buffer (see Note 4).
The antigen capture ELISA is also known as sandwich ELISA and makes use of a "capture" antibody and a "detection" antibody. The capture antibody is coated onto the wells of a microtiter plate before the assay. Then following sample processing, the lysate is incubated in the wells of the microtiter plate. If the sample contains peptides from MERS-CoV (specifically nucleocapsid protein), they will bind with the coated antibody and be "captured" onto the microtiter plate. Even minute amount of viral peptide can be retained in the well if the capture antibody has a high affinity to the peptide and was coated at high concentration. Unbonded proteins are then washed away before the addition of the second, "detection" antibody. The secondary MAb also recognizes the MERS-CoV NP, presumably binds to a distinct epitope, and is conjugated with horseradish peroxidase for detection. The combination of two MAbs in an ELISA assay offers increased sensitivity for MERS-CoV NP. On the other hand, this "sandwich" approach also allows improved specificity for the MERS-CoV nucleocapsid protein by combining the specificities of the two MAbs, allowing it to differentiate and identify MERS-CoV spiked sample from other samples from healthy and patients who contracted various respiratory tract infections, as previously demonstrated [3].

In this assay the nucleocapsid protein was selected as the target for generating antibodies to detect MERS-CoV. According to previous experience when working with SARS-CoV, we observed that the NP is a highly immunogenic and abundantly expressed structural protein, and a more preferable target than the spike (S) protein $[16,17]$. Working with the hypothesis that the NP protein of MERS-CoV might also be a desirable target when developing an antigen capture ELISA for it, we have shown that the assay offers high specificity and sensitivity, as mentioned above. The steps related to the cloning and purification of (His) ${ }_{6}$-tagged 


\subsection{Preparing Solutions}

\subsection{Coating Microtiter Plates with Antibody}

\subsection{Sample Processing}

recombinant NP (rNP) of MERS-CoV for the generation of antiMERS-CoV-rNP MAbs will not be described, as there are commercially available antibodies readily available for purchase. The horseradish peroxidase (HRP) system was used for the colorimetric visualization at the final stage of the assay. Commercial ELISA kits may utilize other detection methods; optimization may be needed. For readers who would like to generate their own HRP conjugated detection antibody, there are also kits available.

When preparing solutions and buffers for the assay, investigators should be aware that "old" buffers may be more likely to be contaminated. The accuracy and reproducibility of the assay can be affected, as the peptides from fungus or other microorganisms may compete with the target antigen. Prepare fresh solutions periodically $(\sim 1$ month $)$; autoclave or filter sterilize the buffers if available. If contaminations are a common occurrence, the addition of $0.05 \%$ sodium azide $\left(\mathrm{NaN}_{3}\right)$ as a preservative is an option.

1. Dilute the MERS-CoV-rNP MAb IF6 in blocking buffer. (see Note 2).

2. Coat the microtiter plates by adding $100 \mu \mathrm{L}$ of the solution prepared per well.

3 . Cover the plate with an adhesive plastic cover and incubate at $37^{\circ} \mathrm{C}$ overnight ( see Note 3 ).

4. Discard the adhesive plastic cover and remove the solution.

5. Wash the plate with $300 \mu \mathrm{L}$ of washing buffer per well for five times using an automatic microplate washer.

6. Dry the plate by patting the plate on a paper towel.

7. Allow the plate to air-dry. Proceed to the next step or cover the plate with adhesive plastic cover and store at $4{ }^{\circ} \mathrm{C}$ until use.

All processes with potentially infectious MERS-CoV materials should be handled according to institutional, local, and international regulations, guidelines, and standard operating procedures (SOP) to avoid spreading and contamination of the facility. All work with infectious MERS-CoV was performed inside a biosafety level-2 cabinet with SOP in approved biosafety level-3 facilities during development and evaluation of the assay $[3,18,19]$.

1. Aliquot $50 \mu \mathrm{L}$ of viral lysis buffer to new $1.5 \mathrm{~mL}$ conical screw cap tubes according to the number of samples and controls (see Note 4).

2. Pipette $100 \mu \mathrm{L}$ of specimen from the sample collection tube to the $1.5 \mathrm{~mL}$ conical screw cap tubes with viral lysis buffer, mix well. Allow sufficient time for inactivation.

3. Transfer the inactivated sample out of the biosafety cabinet to the general laboratory area, according to established SOP. 


\subsection{Sample Dilution and Incubation}

\subsection{Dilution of} Secondary Antibody and Incubation

\subsection{Detection and} Readout
1. Serially dilute the inactivated sample in sample dilution buffer, add $100 \mu \mathrm{L}$ of the mixture into the wells in duplicates.

2. Gently shake the plate for $2 \mathrm{~min}$ to mix well, and then incubate at $37{ }^{\circ} \mathrm{C}$ for $30 \mathrm{~min}$ while being covered with an adhesive plastic cover.

3. Discard the adhesive plastic cover and remove the solution.

4. Wash the plate with $300 \mu \mathrm{L}$ of washing buffer per well for five times using an automatic microtiter plate washer.

1. Dilute the secondary detection antibody (MAb 7C4 conjugated with HRP) in enzyme dilution buffer immediately before use.

2. Add $100 \mu \mathrm{L}$ of the diluted detection antibody to each well using an 8-channel pipette.

3 . Cover the plate with an adhesive plastic cover and incubate at $37^{\circ} \mathrm{C}$ for $30 \mathrm{~min}$.

4. Discard the adhesive plastic cover and remove the solution.

5. Wash the plate with $300 \mu \mathrm{L}$ of washing buffer per well for five times using an automatic microtiter plate washer.

1. Add $100 \mu \mathrm{L}$ of $3,3^{\prime}, 5,5^{\prime}$-Tetramethylbenzidine (TMB) substrate solution to each well ( see Note 5 ).

2. Cover the plate with aluminum foil to protect from light, incubate for $10 \mathrm{~min}$ at room temperature.

3. Add $50 \mu \mathrm{L}$ of stop solution to each well to stop the reaction.

4. Read the plate using an automatic plate reader at wavelength $450 \mathrm{~nm}$.

5. Analyze the data by using the predetermined cutoff value.

\section{Notes}

1. An automated microtiter plate washer-dispenser would be a good addition to the workflow as the washing steps can be performed in a shorter amount of time and with greater consistency. But multichannel or even single-channel pipettes can be used instead.

2. The actual dilution of antibodies depends on the batch and quality of the MAbs used. Evaluations and characterization to determine the specificity and sensitivity have to be performed to establish the optimal dilution for the highest signal-to-noise ratio.

3. Prevent the microtiter plates to dry up by placing the plates in a box with some moist tissue paper laying under while storing in an incubator or on a rack in a warm water bath. 
4. There are many viral lysis buffers available for purchase from bio-reagents vendors, e.g., buffer AL from Qiagen. Readers could request samples and perform their own testing on the conditions required to efficiently inactivate MERS-CoV.

5. TMB solutions are normally purchased from bio-reagents vendors at ready-to-use dilations, follow manufacturer's instructions.

\section{Acknowledgments}

This work is partly supported by the Theme-based Research Scheme (Project No. T11/707/15), University Grant Committee; Consultancy Service for Enhancing Laboratory Surveillance of Emerging Infectious Diseases and Research Capability on Antimicrobial Resistance for Department of Health, HKSAR Government; and University Development Fund, The University of Hong Kong.

\section{References}

1. (WHO), W.H.O., MERS situation update, July 2018. 2018, World Health Organization: World Health Organization, 20 Avenue Appia, 1211 Geneva 27, Switzerland. p. 1

2. (WHO), W.H.O., Laboratory Testing for Middle East Respiratory Syndrome Coronavirus. 2018 (January 2018): p. 8

3. Chen Y et al (2015) A sensitive and specific antigen detection assay for Middle East respiratory syndrome coronavirus. Emerg Microbes Infect 4(4):e26

4. Corman VM et al (2012) Detection of a novel human coronavirus by real-time reverse-transcription polymerase chain reaction. Euro Surveill 17(39):20285

5. Corman VM et al (2012) Assays for laboratory confirmation of novel human coronavirus (hCoV-EMC) infections. Euro Surveill 17 (49):20334

6. Muller MA et al (2015) Presence of Middle East respiratory syndrome coronavirus antibodies in Saudi Arabia: a nationwide, crosssectional, serological study. Lancet Infect Dis 15(6):629

7. Perera RA et al (2013) Seroepidemiology for MERS coronavirus using microneutralisation and pseudoparticle virus neutralisation assays reveal a high prevalence of antibody in dromedary camels in Egypt, June 2013. Euro Surveill 18(36):pii $=20574$
8. Saeidnia S, Abdollahi M (2013) Are other fluorescent tags used instead of ethidium bromide safer? Daru 2l(1):71

9. Drosten C et al (2013) Clinical features and virological analysis of a case of Middle East respiratory syndrome coronavirus infection. Lancet Infect Dis 13(9):745-751

10. Kapoor $\mathrm{M}$ et al (2014) Clinical and laboratory findings of the first imported case of Middle East respiratory syndrome coronavirus to the United States. Clin Infect Dis 59 (11):1511-1518

11. Alagaili AN et al (2014) Middle East respiratory syndrome coronavirus infection in dromedary camels in Saudi Arabia. MBio 5(2): e00884-e00814

12. Chen Y et al (2016) A highly specific rapid antigen detection assay for on-site diagnosis of MERS. J Infect 73(1):82-84

13. Woo PCY et al (2018) Rapid detection of MERS coronavirus-like viruses in bats: potelntial for tracking MERS coronavirus transmission and animal origin. Emerg Microbes Infect $7(1): 18$

14. Lau SK et al (2013) Genetic characterization of Betacoronavirus lineage $\mathrm{C}$ viruses in bats reveals marked sequence divergence in the spike protein of pipistrellus bat coronavirus HKU5 in Japanese pipistrelle: implications for the origin of the novel Middle East respiratory 
syndrome coronavirus. J Virol 87(15): $8638-8650$

15. Yuan Q et al (2011) Differential diagnosis of pandemic (HINl) 2009 infection by detection of haemagglutinin with an enzyme-linked immunoassay. Clin Microbiol Infect 17 (10):1574-1580

16. Lau SK et al (2005) SARS coronavirus detection methods. Emerg Infect Dis 11 (7):1108-1111

17. Lau SK et al (2004) Detection of severe acute respiratory syndrome (SARS) coronavirus nucleocapsid protein in sars patients by enzyme-linked immunosorbent assay. J Clin Microbiol 42(7):2884-2889

18. Chan JF et al (2013) Differential cell line susceptibility to the emerging novel human betacoronavirus 2c EMC/2012: implications for disease pathogenesis and clinical manifestation. J Infect Dis 207(11):1743-1752

19. Chan KH et al (2013) Cross-reactive antibodies in convalescent SARS patients' sera against the emerging novel human coronavirus EMC (2012) by both immunofluorescent and neutralizing antibody tests. J Infect 67(2): $130-140$ 